\title{
Assessment of Microsoft Kinect Technology (Kinect for Xbox and Kinect for Windows) for Patient Monitoring during External Beam Radiotherapy
}

\author{
F. Tahavori, M. Alnowami, J. Jones, P. Elangovan, E. Donovan \& K. Wells
}

\begin{abstract}
In external beam radiotherapy, patient misalignment during set-up and motion during treatment may result in lost dose to target tissue and increased dose to normal tissues, reducing therapeutic benefit. The most common method for initial patient setup uses room mounted lasers and surface marks on the skin. We propose to use the Microsoft Kinect which can capture a complete patient skin surface representing a multiplicity of 3D points in a fast reproducible, marker-less manner. Our first experiments quantitatively assess the technical performance of Kinect technology using a planar test object and a precision motion platform to compare the performance of Kinect for Xbox and Kinect for Windows. Further experiments were undertaken to investigate the likely performance of using the Kinect during treatment to detect respiratory motion, both in supine and prone positions. The Windows version of the Kinect produces superior performance of less than $2 \mathrm{~mm}$ mean error at $80-100 \mathrm{~cm}$ distance.
\end{abstract}

\section{INTRODUCTION}

To optimise radiotherapy treatment and dose delivery, it is necessary to precisely align the patient prior/during each treatment and also to account for motion during treatment delivery, in order to minimise the dose to the normal tissues without compromising therapeutic benefit [1]. For patient setup a variety of technologies are used including portal imaging and range imaging [2]. Spirometry, temperature sensors, markerbased camera tracking and pressure belts may be applied for respiratory motion tracking [3].

By contrast, we propose using a marker-less 3D depth camera, the Microsoft Kinect, available in two versions: Kinect for Xbox, and Kinect for Windows, which can measure the 3D depth distribution of the patient torso surface to potentially deliver greater and faster reproducibility of patient alignment and also for monitoring patient motion during treatment delivery. In this work, we assess the quantitative performance of two versions of the Kinect technology: Xbox and Windows, to compare their performance under controlled conditions. In addition, the use of Kinect to measure respiratory motion was also investigated.

\section{A. Microsoft Kinect Technology}

The Kinect unit includes an RGB colour camera, an IR projector, an IR camera and a multi array microphone. Mi-

F. Tahavori, J. Jones, P. Elangovan and K. Wells are with the CVSSP, FEPS, University of Surrey, Guildford, Surrey GU2 7XH, UK.

M. Alnowami is with the Nuclear Engineering Department, King Abdulaziz University, P.O. Box 80204 Jeddah, Kingdom of Saudi Arabia.

E. Donovan is with the Royal Marsden NHS Trust, Sutton, Surrey SM2 5PT, UK.

E-mail: f.tahavori@surrey.ac.uk.

Acknowledgments: The authors would like to thank Mr Robert Derham and Mr Tom Gibbons in the Department of Physic for help and assistance in the preliminary experiments and Dr Simon Hadfield for useful discussions regarding camera calibration.

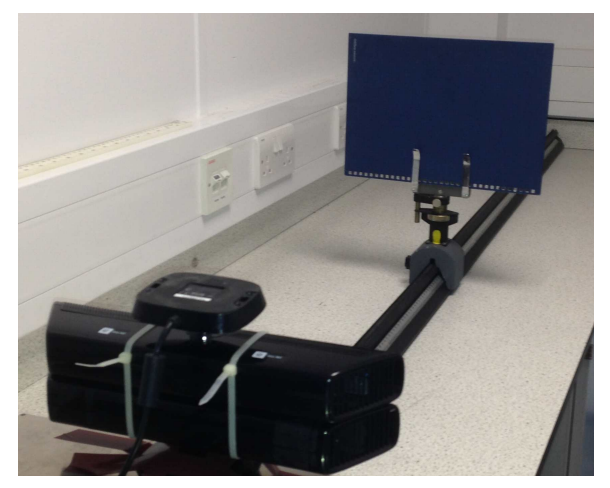

Fig. 1. Both Kinects were mounted on an optical rail that included a calibrated millimeter graticule. A plain card served as a test object and was placed at several different distances from the Kinect units.

crosoft Kinect works with PrimeSense infrared technology [4] wherein an infrared laser projector combined with a monochrome CMOS sensor is used as a depth sensor in order to capture the 3D scene information from a continuously projected infrared light pattern. The resulting depth map shows the distance of a surface from the camera.

In 2012, the Kinect for Windows version was released. The Kinect for Windows sensor can be used in closer proximity to a surface than the Xbox version due to an upgraded firmware. It is claimed that this near-mode feature can localise an object at a closer range of $40-50 \mathrm{~cm}$ compared to Kinect for Xbox which requires a minimum distance of approximately $100 \mathrm{~cm}$.

\section{EXPERIMENTAL EVALUATION TRANSFORMATION PERFORMANCE OF KINECT}

This study focuses for the first time, as far as we are aware on the intrinsic technical performance assessment of Kinect for use in external beam radiotherapy. The technical performance of the Kinect technology was evaluated quantitatively using a planar test object, mounted on an optical rail for gross displacement and a precision motion platform for fine step measurements. Also, further experiments were undertaken to study the performance of using the Kinect to detect respiratory motion initially with a Gail motion controller which has respiratory-like displacement and subsequently real volunteers; investigating respiratory motion both in the supine and prone positions.

\section{A. Test Object Experiments}

To initially compare the performance of Kinect for Xbox and Kinect for Windows under controlled conditions, both units were simultaneously mounted on an optical rail and 


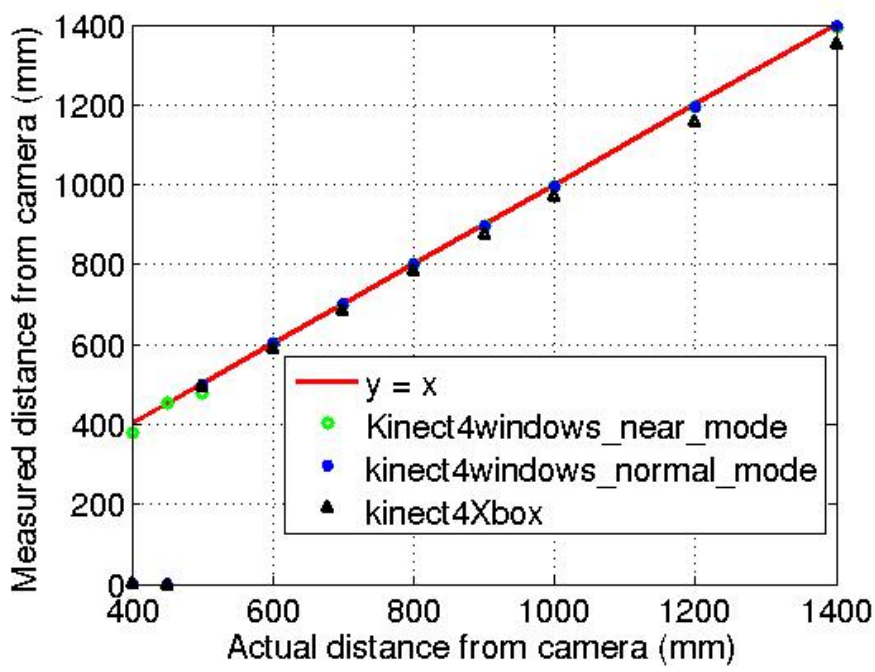

Fig. 2. Kinect for Windows in normal-mode and Kinect for Xbox can not work at a distance range below $50 \mathrm{~cm}$, whilst Kinect for Windows appears to offer superior performance over a range of distances.

a plain test card was placed at a certain distance as a test object (see Figure 1). Data were acquired and analysed using the Microsoft Kinect SDK and Matlab. The performance of both cameras was investigated at different distances and the response was averaged over 1000 frames of depth image data to reduce noise.

1) Gross Step Translation: A test card was placed at varying distances from both Kinect cameras to assess their performances. The card-to-camera distance was varied from $40 \mathrm{~cm}$ to $140 \mathrm{~cm}$. At each measured distance, data were acquired with the Kinect for Windows operating in normalmode and near-mode, in addition to using the Kinect for Xbox unit. In the distance range $40-50 \mathrm{~cm}$ the step size was $5 \mathrm{~cm}$, whilst at a distance range $50-100 \mathrm{~cm}$ and $100-140 \mathrm{~cm}$, the steps size varied by $10 \mathrm{~cm}$ and $20 \mathrm{~cm}$ respectively. The error margin can be estimated by subtracting the distance estimated by the camera from the true measured distance. As can be seen in Figure 2, Kinect for Windows in normal-mode and Kinect for Xbox can not work at a distance range below $50 \mathrm{~cm}$. However Kinect for Windows operating in near-mode appears to have superior performance to both of the above.

This study represents the comparison of both units with data acquired at the same fixed positions. At each distance, depth data were captured using 1000 frames and averaged to decrease noise within the images. In the comparison of the errors of both systems, the Windows version produces superior performance compared with the Xbox version if the target is placed at a distance of $60-140 \mathrm{~cm}$. This draws a focus on undertaking further experiments with Kinect for Windows in normal-mode and near-mode.

2) Fine Step Translation: To investigate the Kinect for Windows performance in more detail a rectangular box comprising solid water equivalent plastic of $20 \mathrm{~cm} \times 20 \mathrm{~cm} \times 5 \mathrm{~cm}$ size was placed on precision motion platform. Then at distances of $80 \mathrm{~cm}$ and $100 \mathrm{~cm}$ the box was moved precisely away from the Kinect unit with the motion controller in $2 \mathrm{~mm}, 3 \mathrm{~mm}$, $5 \mathrm{~mm}$ and $10 \mathrm{~mm}$ steps to examine displacement performance in detail. Data were acquired with the Kinect for Windows operating in normal-mode and near-mode at each measured distance. To determine the error margin at each distance, the depth image was averaged over 1000 frames. The averaged depth data was then converted to point cloud format and real world dimension by using calibration matrix. Real world coordinates $\left(x_{r}, y_{r}, z_{r}\right)$ and pixel coordinates $\left(x_{p}, y_{p}, 1\right)$ are related by Equation 1, where $z$ is depth pixel value at pixel $\left(x_{p}, y_{p}\right)$ and $K$ is camera matrix given by Equation 2. $f_{o}$ and $c c$ are focal length and principal point respectively.

$$
\begin{gathered}
{\left[\begin{array}{l}
x_{r} \\
y_{r} \\
z_{r}
\end{array}\right]=z \times K^{-1} \times\left[\begin{array}{c}
x_{p} \\
y_{p} \\
1
\end{array}\right]} \\
K=\left|\begin{array}{ccc}
f_{o 1} & 0 & c c_{1} \\
0 & f_{o 2} & c c_{2} \\
0 & 0 & 1
\end{array}\right|
\end{gathered}
$$

Finally the point cloud was registered to the reference model using ICP with 6 degrees of freedom (DOF) [5], [6] combined with RANSAC [7], [8]. Figure 3 shows the error between the Kinect units' estimated distance and the actual distance in $\mathrm{mm}$ in distances $80 \mathrm{~cm}$ and $100 \mathrm{~cm}$ for both Kinect for Windows in normal-mode and Kinect for Windows in near-mode and also the effect of combining ICP and RANSAC to reduce the effect of outliers in the data. As can be seen in Figure 3, Kinect for Windows in near-mode has an error less than $1 \mathrm{~mm}$. Thus, using ICP with RANSAC produces lower levels of error.

3) Rotation Transformation: To analyse the accuracy of target realignment using Kinect technology, a rectangular test object was captured by Kinect for Windows in both normalmode and near-mode. After acquisition of the test object surface as a reference model, the object was rotated by a known rotation. Then, the test object surface was captured again. At each measured angle, the depth depth image was averaged over 1000 frames and in the same way as translation experiments, the averaged depth data was converted to point cloud and real world dimension by calibration matrix. Also, the point cloud was registered to the reference model using ICP and RANSAC. The accuracy of Kinect units was estimated by the difference between rotation angles obtained by Kinect and the known defined rotation while the distance between test object and Kinect unit was about $100 \mathrm{~cm}$. Table I shows the Kinect units' estimated angle and the actual angle in degree in distances $100 \mathrm{~cm}$ from Kinect operating in both near-mode and normalmode. As can be seen in Table I, Kinect for Windows in nearmode has error less than a 1 degree compared with Kinect for Windows in normal-mode at this distance.

\begin{tabular}{ccc} 
Ground truth & normal-mode & near-mode \\
\hline $3^{\circ}$ & $1.4^{\circ}$ & $2.5^{\circ}$ \\
$4^{\circ}$ & $2.4^{\circ}$ & $4.8^{\circ}$ \\
$7^{\circ}$ & $3.6^{\circ}$ & $6.8^{\circ}$
\end{tabular}

TABLE I

THE KINECT FOR WINDOWS IN NORMAL-MODE AND NEAR-MODE ROTATION'S RESULTS AND THE KNOWN DEFINED ROTATION. ACQUIRED AS AN AVERAGE OF 1000 FRAMES AT DISTANCE OF $100 \mathrm{CM}$.

\section{B. Head Phantom (Rando) Rotation Experiments}

A further experiment was performed to assess the accuracy of head phantom realignment. In doing so, a Rando head phantom was placed on motion platform and was captured 


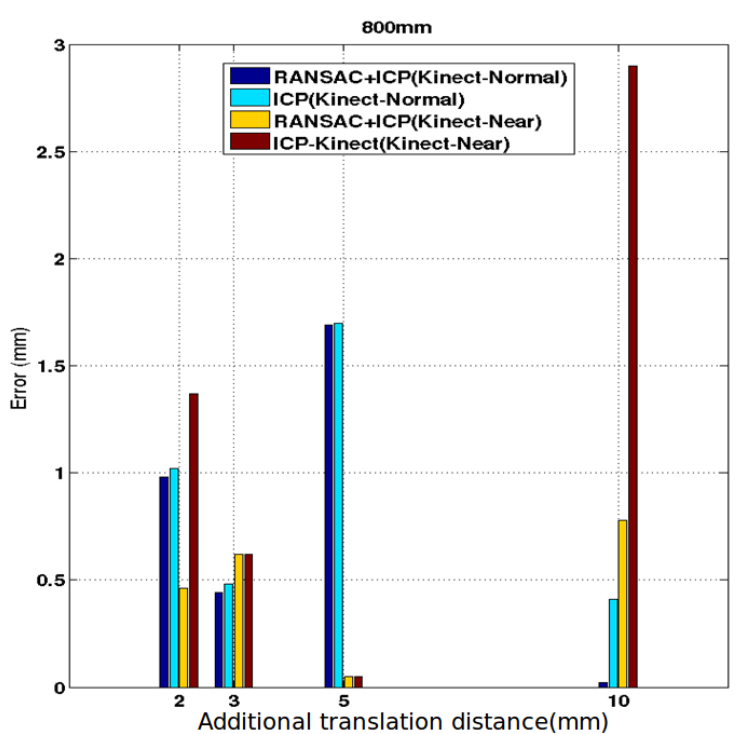

(a)

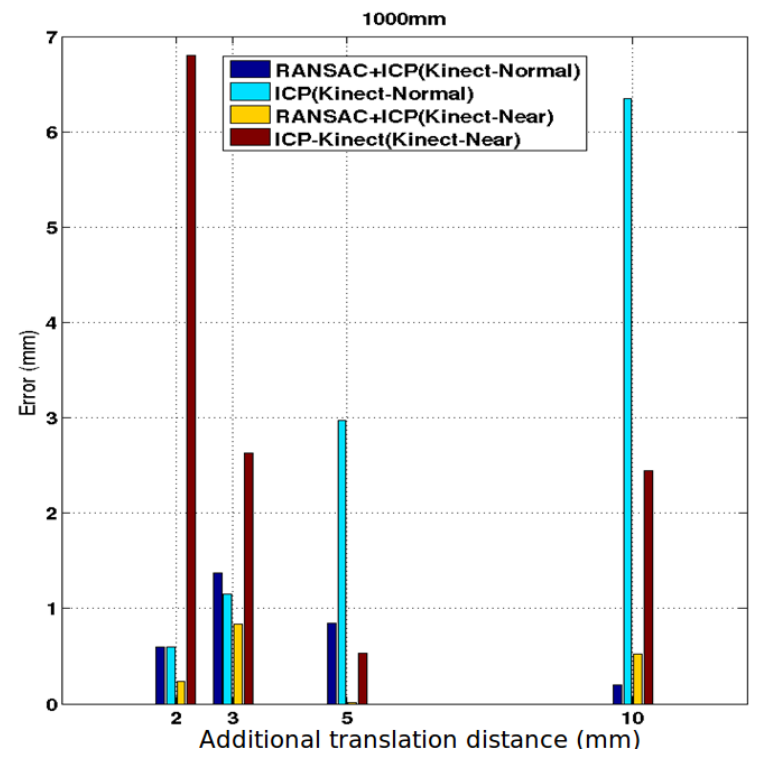

(b)

Fig. 3. The error between Kinect for Windows units' estimated distance and the actual distance in $\mathrm{mm}$. Kinect for Windows in near-mode gives errors less than $1 \mathrm{~mm}$ for distance $100 \mathrm{~cm}$

by Kinect while the distance between the head phantom and Kinect was about $80 \mathrm{~cm}$. Figure 4(a) shows the experimental arrangement and its corresponding depth image 4(b).

After acquisition of the object as a reference model, the object was rotated by a known rotation as 2, 3, 4, 5 and 7 degrees. Then, the Rando phantom was captured again for 1000 frames and averaged to reduce noise at each position. After the depth data was converted to point cloud and real world dimensions, the point cloud was registered to the reference model using ICP. Figure 5 illustrates the RMS error between the angle calculated using ICP with the Kinect point cloud data and the actual angle in degrees at $80 \mathrm{~cm}$.

\section{EXPERIMENTAL EVALUATION METHOD FOR RESPIRATORY MOTION TRACKING}

Given the performance of Kinect technology shown in section II, we proposed using the Kinect for monitoring

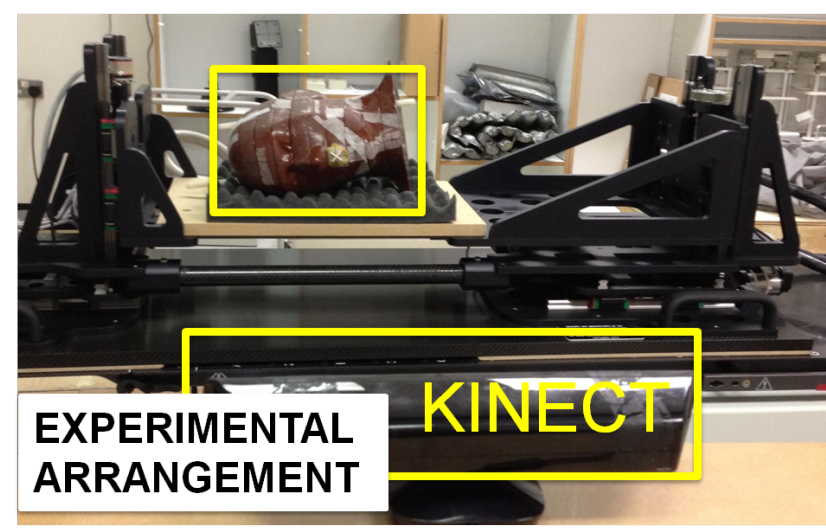

(a)

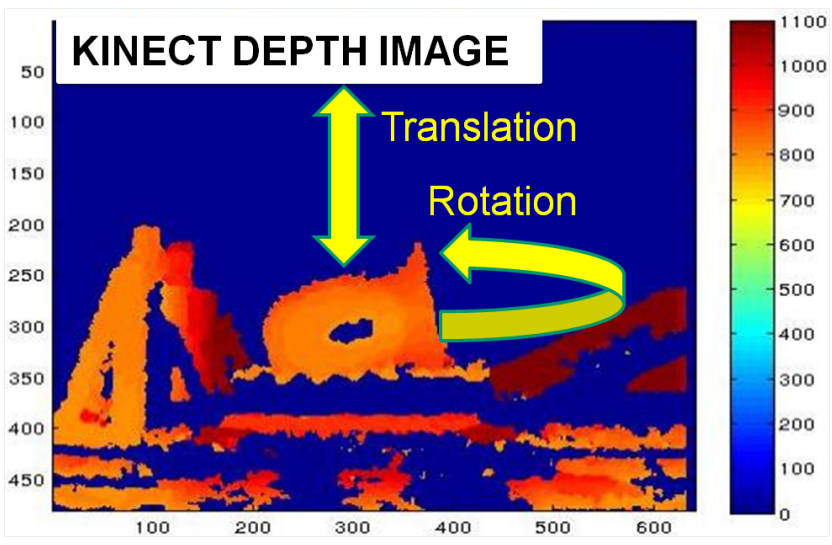

(b)

Fig. 4. (a) Experimental arrangement: A Rando head phantom was placed on the motion platform as shown in box by yellow and captured by Kinect for Windows in normal-mode and near-mode. (b) Example of an obtained depth map.

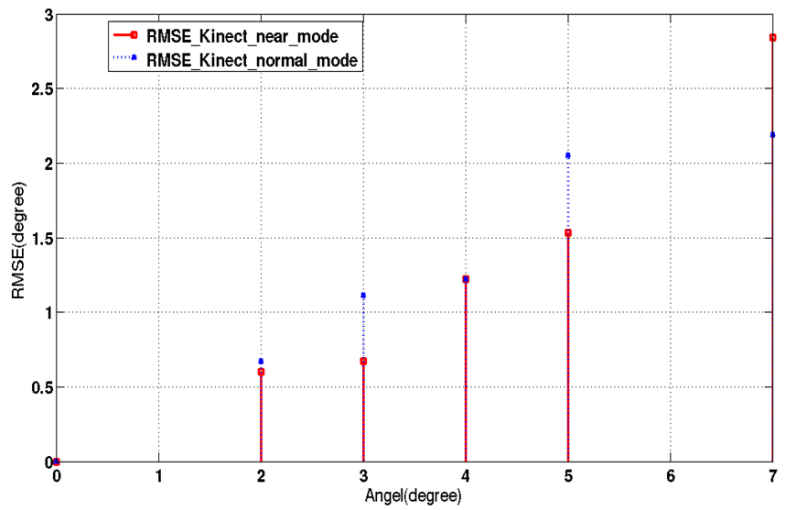

Fig. 5. The RMS error between the Kinect and the actual angle in degree at $80 \mathrm{~cm}$ for Kinect for Windows in normal-mode and near-mode.

respiratory motion monitoring both in the supine and prone positions.

\section{A. Synthesis Motion Controller Semi-respiratory Movement Monitoring}

To study the performance of kinect for Windows in nearmode and normal-mode for respiratory motion tracking, respiratory-like motion was created by a precision motion controller. A solid plastic block with size of $20 \mathrm{~cm} \times 20 \mathrm{~cm}$ $\times 5 \mathrm{~cm}$ was placed on the motion platform while the distance between the Kinect and block was about $100 \mathrm{~cm}$. Figure 6 


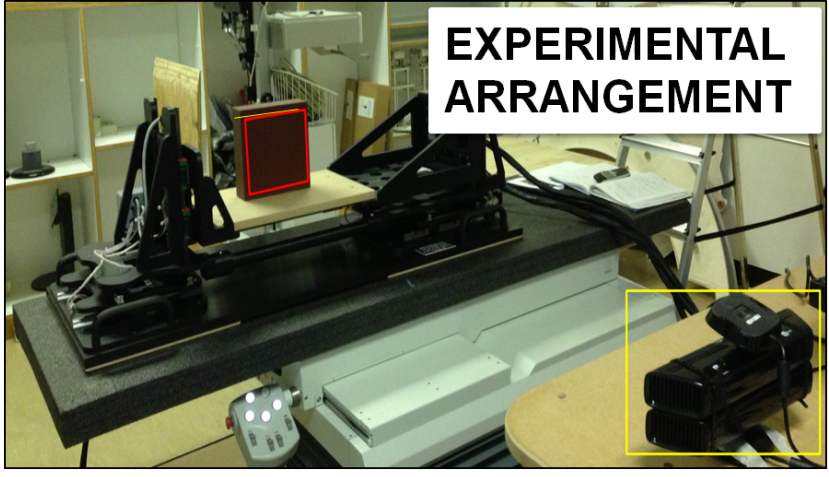

(a)

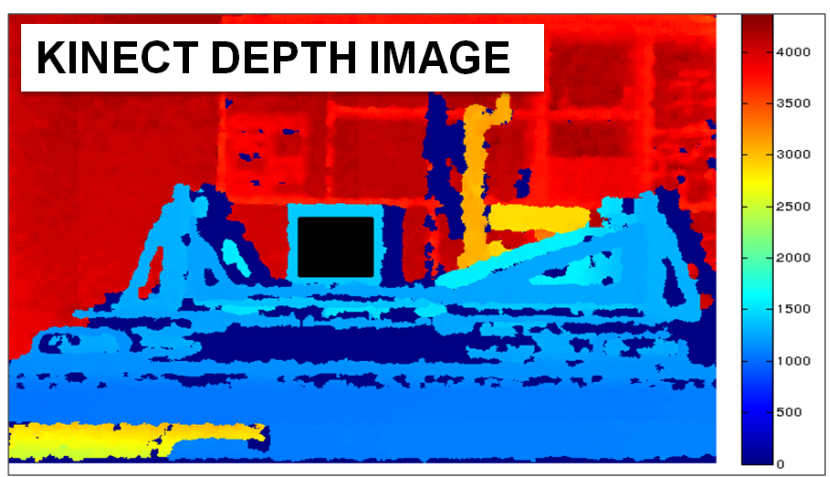

(b)

Fig. 6. (a) Experimental setup: A block served as a test object and was moved by Galil motion controller (DMC 4060). (b) Regions of interest shown in black, overload over depth map.

shows the experimental arrangement and its Kinect depth image.

The test object was moved according to Lujan motion [9] in the $\mathbf{z}$ direction. The motion platform has an accuracy of $0.2 \mathrm{~mm}$ and was displaced according to Equation 3 by a Galil DMC-4060 motion controller [10] to examine how the Kinect can measure known respiratory-like motion:

$$
x(t)=10 \cos ^{6}(\pi t) / \tau+\pi / 2
$$

The error was obtained by subtracting the motion controller displacement from the motion captured by Kinect. Figure 7 illustrates the Kinect captured movement and actual controller displacement in red and green respectively. The mean error was approximately $0.57 \mathrm{~mm}$.

\section{B. Real volunteers Respiratory Motion Monitoring}

1) Prone Position: In the first experiment a volunteer lay prone on a treatment couch with the Kinect for Xbox placed at $1 \mathrm{~m}$ from the midpoint of the spine. Figure 8(a) shows the experimental arrangement and Figure 8(b) represents dynamic data acquired for both tidal and deep breathing in the prone position using a mid-thoracic ROI.

2) Supine Position: In the second experiment a volunteer was placed in the supine position and normal tidal breathing was recorded using a Kinect for Windows unit placed at $80 \mathrm{~cm}$ from the volunteer's chest. A color-coded depth map is shown in Figure 9 (top) which also shows the position of 2 ROIs used on thoracic and abdominal surfaces. Figure 9 (bottom) compares tidal breathing motion in the thoracic region compared to the abdominal region of ROIs $\mathrm{A}$ and $\mathrm{B}$

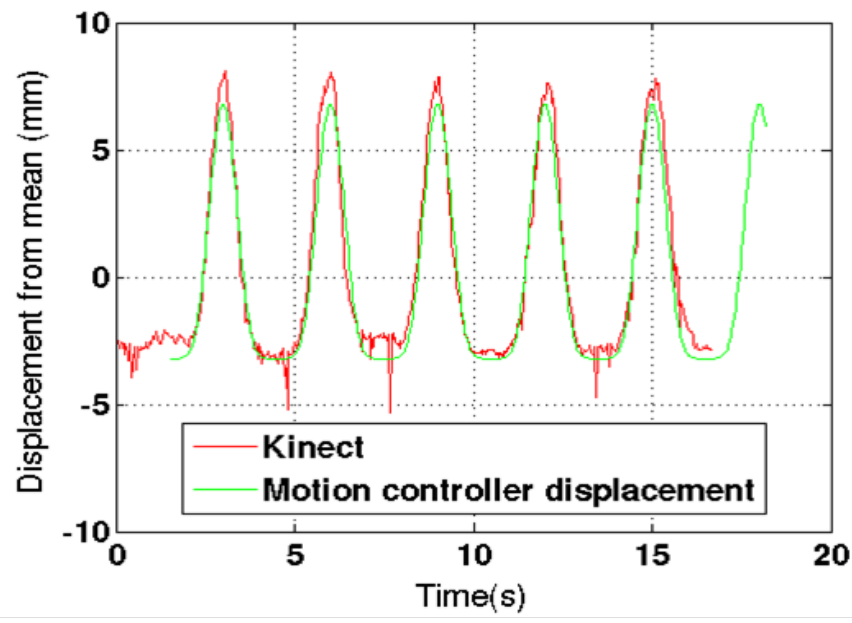

Fig. 7. The dynamic error obtained for respiratory-like breathing in the block placed on motion controller using Kinect for Windows. The maximum error was $2.13 \mathrm{~mm}$, with a mean error of $0.57 \mathrm{~mm}$.

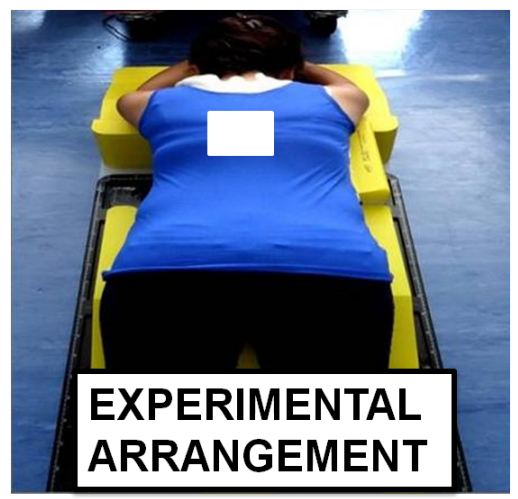

(a)

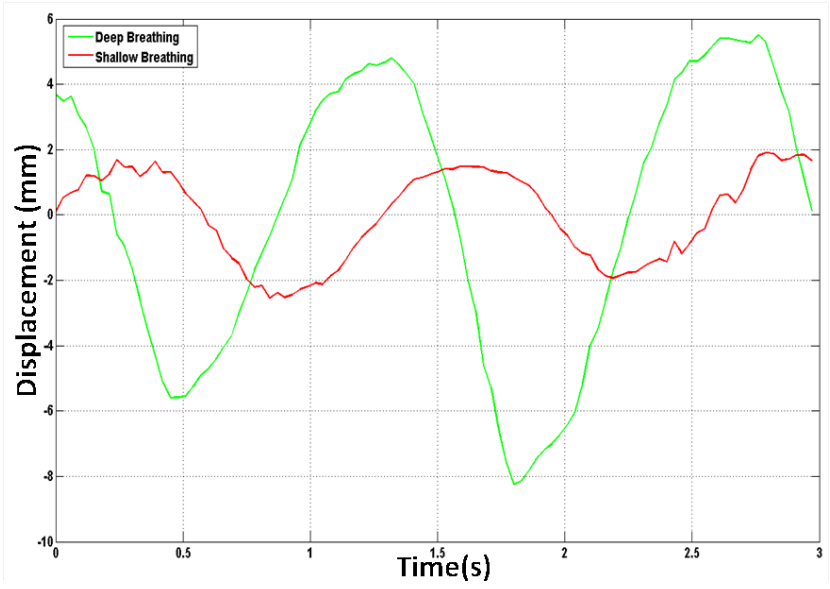

(b)

Fig. 8. (a) Experimental arrangement: A volunteer was positioned in prone position and captured by Kinect for Xbox and region of interest on was obtained as shown in box by white. (b) Shows dynamic data acquired for both tidal and deep breathing in the prone position using a mid-thoracic ROI.

respectively. The displacement in the first cycle demonstrates a deliberate cough. These results suggest that Kinect technology may be useful for monitoring forced as well as tidal breathing, and thus may be useful for gating during dose delivery. 

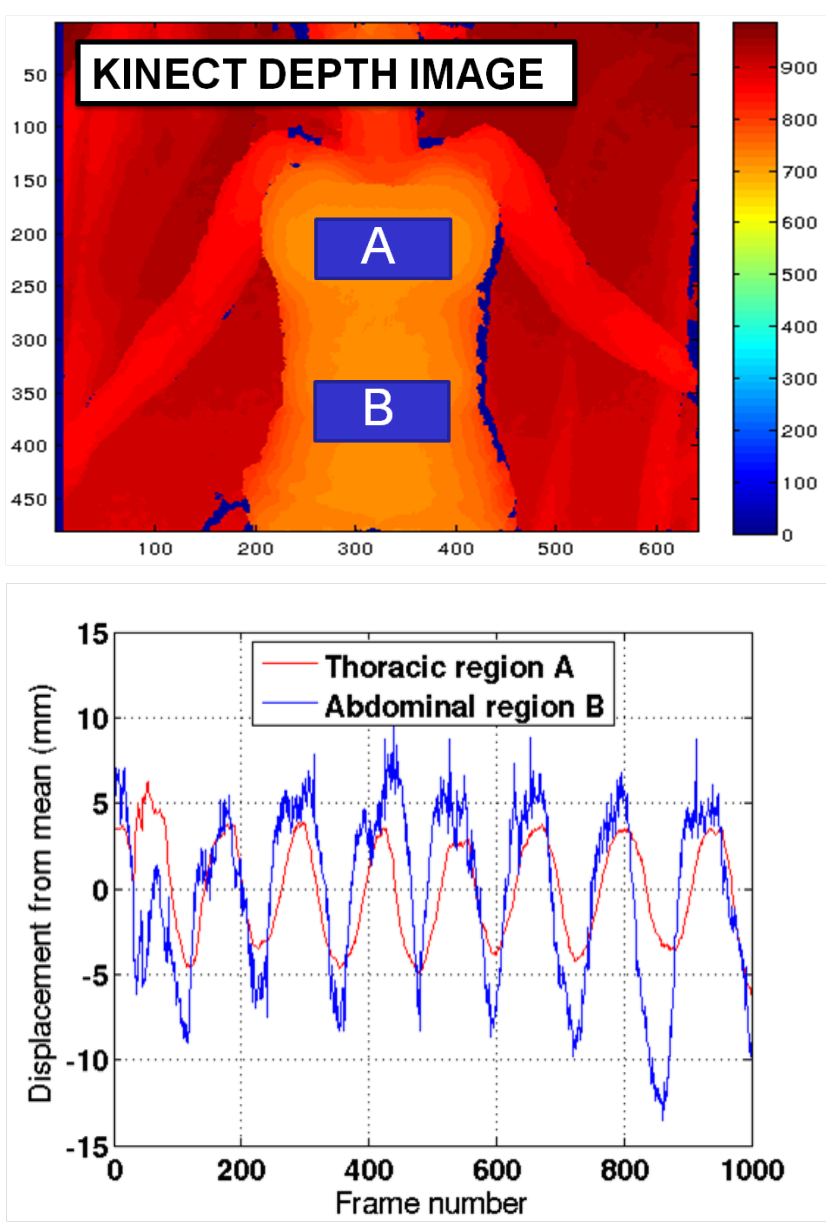

Fig. 9. (top) Depth image of volunteer showing ROIs. (bottom) Respiratory motion plot captured using Kinect for ROIs A and B.

\section{Discussion AND CONCLUSION}

Microsoft Kinect technology, in particular Kinect for Windows, appears to offer a promising non-contact, non-marker based approach for use in external beam radiotherapy, with errors of few mm over a wide range of distances. Furthermore, Kinect for Windows in near-mode results in superior performance compared to Kinect for Windows in normal-mode and Kinect for Xbox (less than a $1 \mathrm{~mm}$ error for static objects, $0.57 \mathrm{~mm}$ for respiratory-like motion) and has a potential for use in external beam radiotherapy. In addition, Kinect may be used for monitoring patient respiration in both the supine and prone position. The device can capture both tidal and deep breathing. It could also act as an external surrogate signal when considering respiratory motion modelling [11], [12].

\section{REFERENCES}

[1] Marco and G. Loi, "Reproducibility of patient setup by surface image registration system in conformal radiotherapy of prostate cancer," Radiation Oncology 4, pp. 9+, feb 2009.

[2] P. J. Schöffel, W. Harms, G. Sroka-Perez, W. Schlegel, and C. P. Karger, "Accuracy of a commercial optical 3D surface imaging system for realignment of patients for radiotherapy of the thorax," Physics in Medicine and Biology 52(13), p. 3949, 2007.

[3] S. Hughes, J. McClelland, S. Tarte, D. Lawrence, S. Ahmad, D. Hawkes, and D. Landau, "Assessment of two novel ventilatory surrogates for use in the delivery of gated/tracked radiotherapy for non-small cell lung cancer," Radiotherapy and Oncology 91(3), pp. 336-341, 2009.

[4] PrimeSense, "Primesense 3D sensor data sheet." http://www.primesense.com/en/press-room/resources/file/4-primesensordata-sheet, 2012.
[5] J. Jones, E. Lewis, M. Guy, and K. Wells, "A virtual dissection based registration to model patient-specific respiratory motion," in IEEE Nuclear Science Symposium Conference 2009, IEEE Nuclear Science Symposium Conference Record, pp. 3571-3576, (Orlando, FL), 2009. Jones, John Lewis, Emma Guy, Matthew Wells, Kevin.

[6] J. Jones, E. Lewis, A. A. Rahni, V. Ezhil, K. Wells, and Ieee, "Mosaics of polynomial transformations giving a patient specific registration to reduce breathing motion artefacts," in 2011 Ieee Nuclear Science Symposium and Medical Imaging Conference, IEEE Nuclear Science Symposium Conference Record, 2011.

[7] M. Zuliani, "RANSAC toolbox for matlab." [web page] http://www.mathworks.com/matlabcentral/fileexchange/18555, Nov. 2008.

[8] K. Lebeda, J. Matas, and O. Chum, "Fixing the locally optimized RANSAC," pp. 1013-1023, BMVA, September 2012.

[9] A. E. Lujan, E. W. Larsen, J. M. Balter, and R. K. Ten Haken, "A method for incorporating organ motion due to breathing into 3D dose calculations," Medical Physics 26(5), pp. 715-720, 1999.

[10] G. A. Davies, P. Clowes, J. L. Bedford, P. M. Evans, S. Webb, and G. Poludniowski, "An experimental evaluation of the agility MLC for motion-compensated VMAT delivery," Physics in Medicine and Biology 58(13), p. 4643, 2013.

[11] F. Tahavori, M. Alnowami, and K. Wells, "A comparison between adaptive kernel density estimation and gaussian mixture regression for realtime tumour motion prediction from external surface motion," in Nuclear Science Symposium and Medical Imaging Conference (NSS/MIC), 2012 IEEE, pp. 3902-3905, 2012.

[12] R. L. Smith, "Recursive Bayesian Estimation for Respiratory Motion Correction in Nuclear Medicine Imaging," in IEEE NSS/MIC Conf Rec, pp. 2942-2945, 2012. 